\title{
Automatic Video Surveillance System for Pedestrian Crossing Using Digital Image Processing
}

\author{
C. Thirumarai Selvi' and J. Amudha ${ }^{2}$
}

'Department of Electronics \& Communication Engineering, Sri Krishna College of Engineering and Technology, Coimbatore - 641008, Tamil Nadu, India; thirumaraiselvi@skcet.ac.in

${ }^{2}$ Department of Electrical and Electronics Engineering, Dr. Mahalingam College of Engineering and Technology, Pollachi - 642003, Tamil Nadu, India; a_jeyaprakash@yahoo.com.sg

\begin{abstract}
Objective: This work proposes a novel technique to assist the physically challenged people to cross the road near the traffic signal or in the pedestrian crossing with the help of automatic video surveillance. Methods/Statistical Analysis: As per the Indian roadway corporation it is decided to minimum $1.8 \mathrm{~m}$ (width) $\times 2.2 \mathrm{~m}$ (height) is allocated for walking zone. $1800 \mathrm{~mm}$ width is reserved for wheel chair movement in pedestrian. Detection of moving objects in a stable place needs the high security level. Findings: Image processing segmentation algorithm plays an important role to track the moving object in the fixed pedestrian crossing which can assist the physically challenged people or the elderly person. Various morphological filtering operations enhance the quality of segmenting the moving person in the video. This methodology employs histogram of Gaussian detection and object detection is done. The proposed work improves it results by $0.71 \%$ accuracy. Applications/Improvements: This work helps the physically challenged to cross the pedestrian in a safe manner and helps for autonomous vehicles.
\end{abstract}

Keywords: Image Processing, Morphological Operations, Pedestrian Crossing, SVM Classification, Video Surveillance

\section{Introduction}

Surveillance cameras are placed in shopping mall, ATM machine, public places, industries, bank, educational institution, hospitals, traffic signal and others. Static back ground, moving objects, appearance of static and dynamic components of the scene and moving objects are the three main components of the scene. With the advent of video coding technologies, video surveillance is a blooming field for object tracking. Video surveillance with human is a very time consuming process. Hence machine has to analyze the video and extract the necessary information for further application. Many soft computing algorithms have the capacity to automatically detect various objects such as person, vehicle, animals, and equipment etc. Analyze or observe a particular place for business and safety purpose is called as video surveillance. The significance of this system is to help the physically challenged people and elders to cross the traffic signal very safety. Video surveillance importance is applicable in multiple fields known as missile tracking, security purpose, medical laparoscopy, moving robot design during the building crash, road and forest accident prevention etc.

Object tracking is the main sequence used for the video surveillance system. Figure 1 explains the general block diagram of the video surveillance method. Frame difference method, optical flow method and background subtraction methods are the significant methods applied for object tracking. Optical flow method is little bit complex and cannot be used in real time application. Among the various methods, background subtraction method is popularly efficient method selected for the work. 


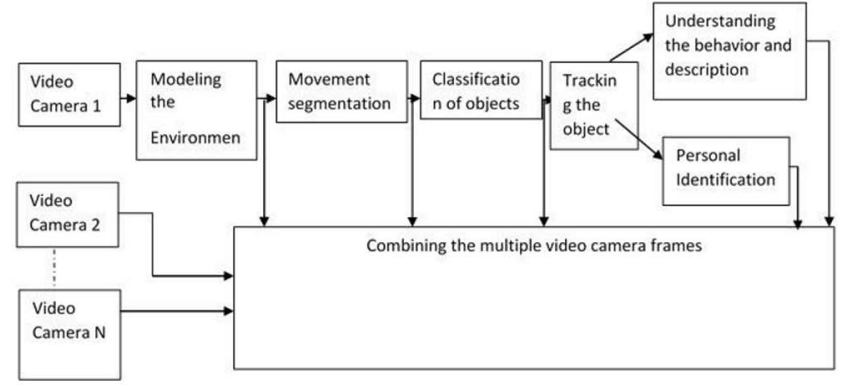

Figure 1. Video surveillance general frame work.

This study contributes algorithms for monitoring pedestrian crossings and identifies the human or vehicle and other objects. With the trained template matching, the activities are analyzed using image processing techniques for segmentation and behavior detection. To monitor this multiple video cameras are installed and data are collected. And this system will work for automated vehicle, and to prevent accidents for other vehicles. The study is organized as follows. Literature survey has been presented in section 2. Section 3 presents the details of existing method. The proposed methodology is discussed in section 4. Section 5 gives the experimentation results with comparison of existing method. Finally section 6 summarizes the work performed.

\section{Materials and Methods}

The research describes a zebra crossing detection ${ }^{1}$ system for operating an intelligent vehicle. This work adapts a morphological filter followed by horizontal projection to extract zebra crossing regions. Then self-similarity recognition method is employed to segment the zebra crossing region. This work achieves an accuracy of $98.5 \%$. But this system, fails to monitor the traffic signal and its control operations. The average time taken to capture one frame is about $57.24 \mathrm{Ms}$. The road scene segmentation process ${ }^{2}$ has developed using convolutional neural network. This $\mathrm{CNN}$ extracts the road scene from the $3 \mathrm{D}$ scene. The developed algorithm generates trained labels from general image data set. From the trained labels, the real time road scenes can be compared and analyzed to understand the road images. This work combines online images with off line images. Compared to the baseline image analysis methods, there is a $7 \%$ improvement of accuracy in the noisy image.

The recent work ${ }^{3}$ for vehicle based mobile mapping system for automatic pedestrian crossing. The preprocessing methods includes projection filtering, monocular vision and contour information analysis. This system gains high recall rate, robustness and precision. The given model can be working in the different situation and light conditions. SVM machine learning algorithm is uses to avoid false identification. The main benefit of this system is to detect perfectly even in defilement and impairment traffic situation of roads. Identification of pedestrians has been done in the work ${ }^{4}$ by combining motion information with image intensity information. The detector employed with Adaboost neural network algorithm to detect a walking person as well as motion appearing information. This study work efficiently, even in the low resolution images such as rain or snow environments. For preprocessing it utilizes a simple rectangular filter with minimum extraction time of $0.25 \mathrm{~ms}$ to handle $360 \times 240$ image size.

A novel method ${ }^{5}$ for detecting the pedestrian by combing HOG and optical flow method. Caltech and ETHZ pedestrian benchmark images are applied for analysis and provide low false positive rates. This automatic pedestrian detection system also gives low average miss rates ranges. Two classifier models named as SVM and Ada-boost methods are used for classification. A data driven approach ${ }^{6}$ has been developed to detect zebra crossings to examine the aerial images. HOG and LBPH methods worked together with SVM algorithm is employed here. This work can contribute a navigational guidance for visually impaired people.

\section{Existing Work}

A part of the existing work was devoted to collect relevant videos of pedestrians crossing near the zebra crossings. This work ${ }^{7}$ investigates the features of pedestrian detection and behavior analysis. The behavior analysis includes, eye gaze, face expression, pose, body motion and hand movements. Advance machine learning techniques are used to solve these tasks. Figure 2 explains the work flow of existing method.

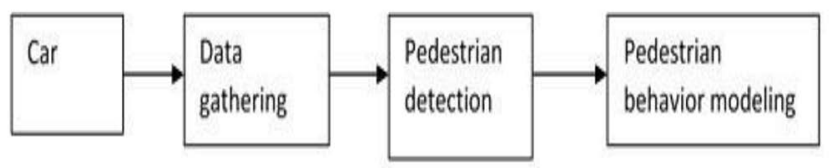

Figure 2. Block diagram of the existing work. 


\section{Methodology}

The proposed work in Figure 3 collects the data from video and separates into different frames. For every frame the back ground can be subtracted from fore ground extraction and compares the foreground for every frame. Histogram of gradient method is useful to create the histogram of different objects for different gradients. The extracted foreground objects are classified using both Support vector machine and Kande algorithm using computer vision. The classified objects based on activities it is tracked for different velocity or speed.

One of the efficient methods useful for object identification is Histogram of oriented gradients (HoG). Using this local object identification and shape has been estimated using histogram. Initially the centered and horizontal gradients are recognized without smoothing. Then the magnitude and gradients are computed. Table 1 lists the different features for identifying the objects for segmentation. Table 2 is used for first level of object recognition for the first level of searching. After the image classification, the second search for classification the Table 3 lists the group of keywords. Table 4 gives the nature of pedestrians who may use the pedestrian. The theoretical walking speed for the ordinary signaling is

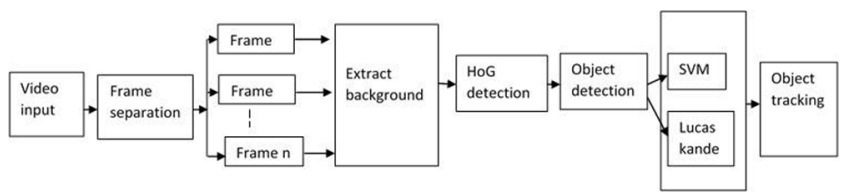

Figure 3. Block diagram of the proposed work.

Table 1. Case structure considered for the proposed algorithm

\begin{tabular}{ll}
\hline \multicolumn{2}{c}{ Case structure } \\
\hline Feature & Positive values \\
Traffic flow & Low, Normal, High \\
Vehicle detected & True, False \\
Speed of detected vehicle & null, Slow, Normal, Fast \\
$\begin{array}{l}\text { Number of positive } \\
\text { intentions }\end{array}$ & Few, Normal, High \\
Time waited & Short, Medium, Long \\
Type of pedestrian & SlowGroup, Slow, Normal, Fast \\
$\begin{array}{l}\text { Proposed transition time } \\
\text { (solution) }\end{array}$ & $\begin{array}{l}\text { null, Extra-Extra time, Extra } \\
\text { time, Normal, Less time, Less- } \\
\text { less time }\end{array}$ \\
\hline
\end{tabular}

Table 2. List the objects used for classification

\begin{tabular}{lll}
\hline Pedestrian & Classification & Visually impaired \\
& recognition & Blind \\
& & Bicycle \\
& Vehicle \\
& Wheel chair \\
\hline
\end{tabular}

Table 3. List of activities for second search

\begin{tabular}{lll}
\hline Pedestrian & Detection recognition & Action \\
& Activity \\
& Gesture \\
& Gait \\
& Behavior \\
\hline
\end{tabular}

Table 4. Types of pedestrians

\begin{tabular}{ll}
\hline Adults & Disabled \\
\hline Elderly & Injured \\
Children & Visually impaired \\
Groups of children & Bicyclists \\
People carrying heavy object & \\
\hline
\end{tabular}

about 1.2 meter per second and for the fat walking it is given by 2 meter per second collected from Handbook 048 from the NRPA for pedestrians. Machine learning duplicates the physical activities of human behavior by electronically perceiving and understanding an image.

\subsection{Feature Extraction}

Features are extracted as sub windows of a sample image. The base size of sub image is kept as $24 \times 24$. Four features are scaled and shifted in all possible combinations. Figure 3 represent the four different feature blocks. Figure 4 specifies the subsamples considered for features.

\subsection{Algorithmic Steps of Feature Learning}

- Learn a single simple classifier

- Classify the data

- Look at where it makes errors

- Reweight the data so that the inputs where we made errors get higher weight in the learning process

- Now learn a $2^{\text {nd }}$ simple classifier on the weighted data

- Combine the $1^{\text {st }}$ and $2^{\text {nd }}$ classifier Figure $5 \& 6$ and weight the data according to where they make errors

- Learn a $3^{\text {rd }}$ classifier Figure 7 on the weighted data and so on until we learn $\mathrm{T}$ simple classifiers 
- Final classifier Figure 8 is the combination of all $\mathrm{T}$ classifiers

- This procedure is called "Boosting" - works very well in practice.

A
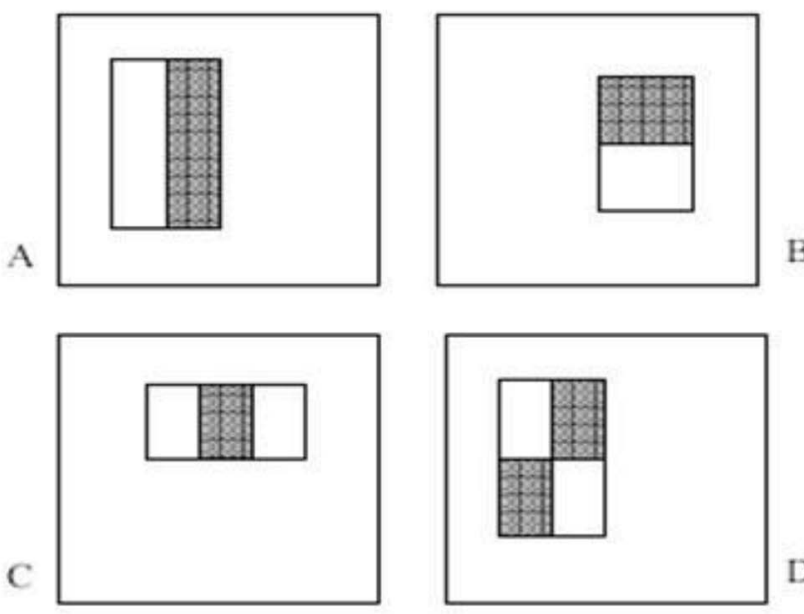

Figure 4. Four different samples for feature extraction.

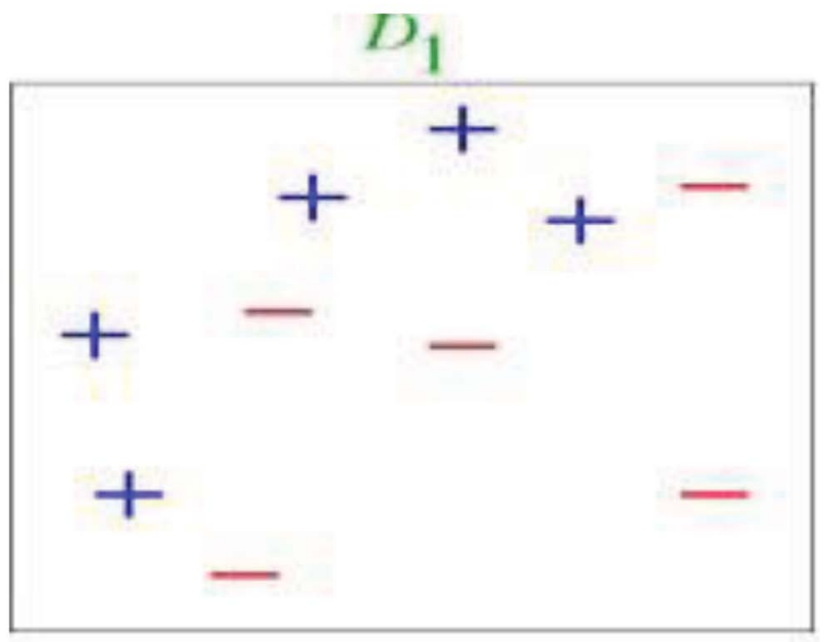

Figure 5. Boosting.

\section{Experiment Results and Discussions}

The experiment is simulated using Matlab and the resulted are shown in Figure 9. Table 5 shows the comparative results of the existing method with the proposed method. The results show that the proposed method has improved accuracy by $0.71 \%$. Also it shows there is a reduce time for classification against the existing
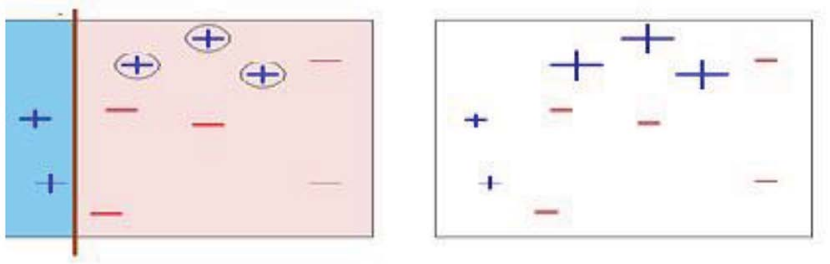

Figure 6. First classifier.
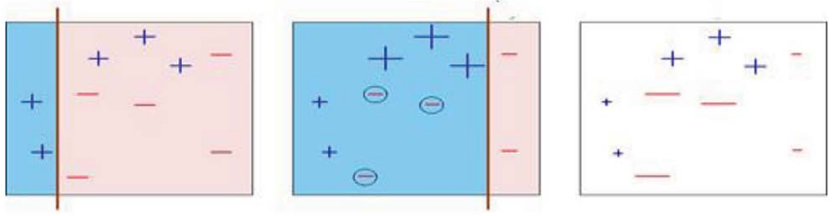

Figure 7. $2^{\text {nd }}$ classifiers.

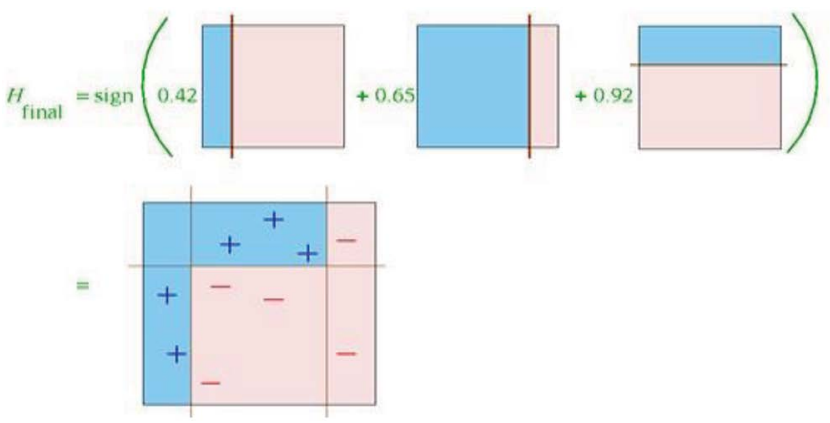

Figure 8. Final Classifier learned by Boosting.

Table 5. Comparison between proposed and existing methods

\begin{tabular}{lcccccc}
\hline & \multicolumn{2}{c}{ Distance. } & \multicolumn{2}{c}{ Velocity } & \multicolumn{2}{c}{ Timing } \\
Features & Existing & Proposed & Existing & Proposed & Existing & Proposed \\
\hline Case1 & 96.71 & 97.03 & 211.54 & 213.31 & 0.456 & 0.441 \\
Case2 & 97.23 & 97.54 & 214.63 & 216.22 & 0.456 & 0.441 \\
Case3 & 97.66 & 97.86 & 211.62 & 211.65 & 0.456 & 0.441 \\
Case4 & 99.02 & 99.50 & 209.10 & 210.49 & 0.456 & 0.441 \\
Case5 & 99.11 & 99.83 & 205.92 & 205.98 & 0.456 & 0.441 \\
\hline
\end{tabular}




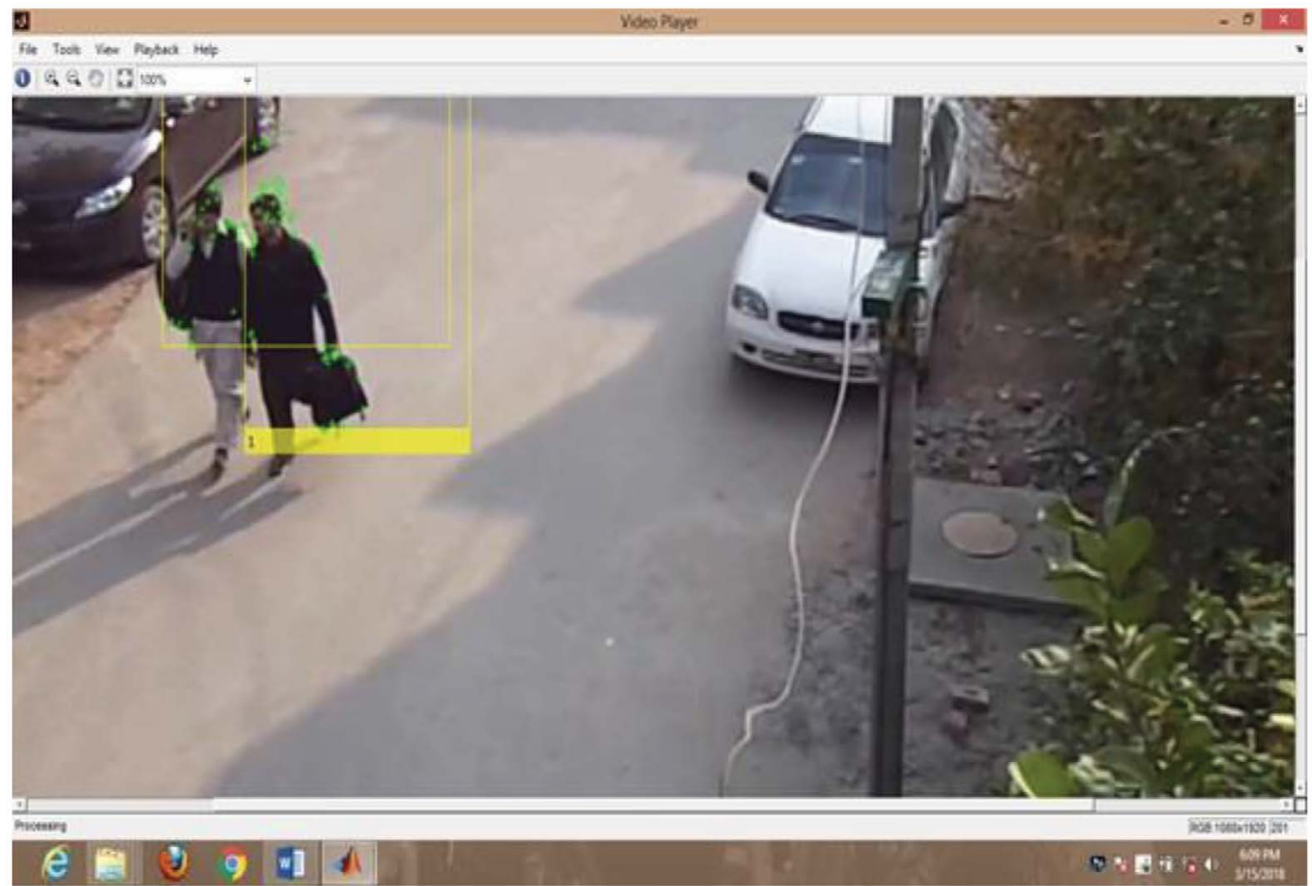

Figure 9. Object tracking results.

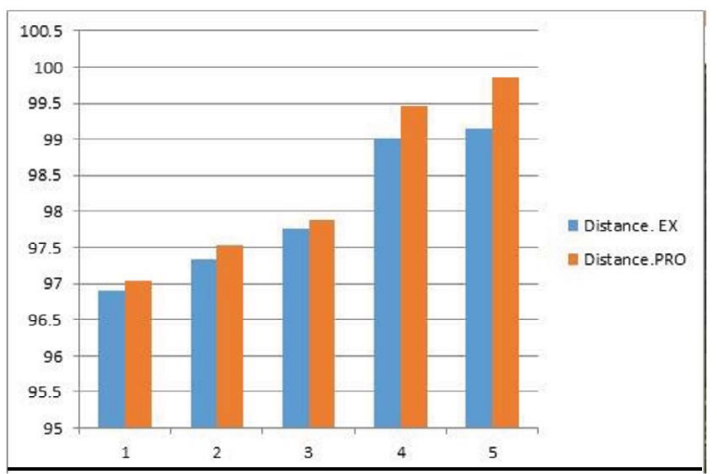

Figure 10. Distance between previous method and existing method.

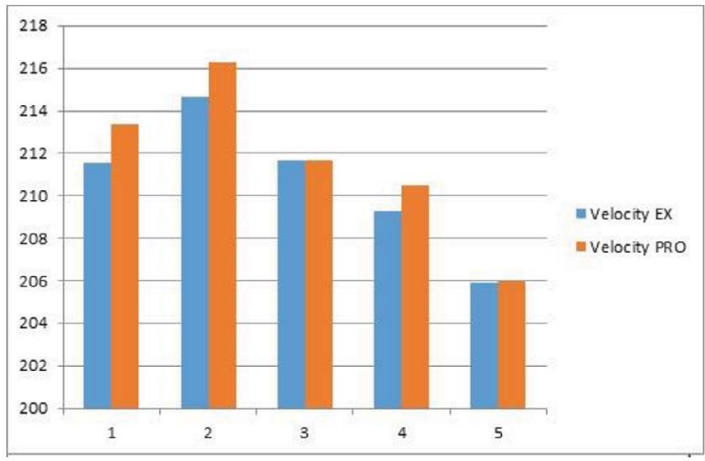

Figure 11. Velocity between previous method and existing method.

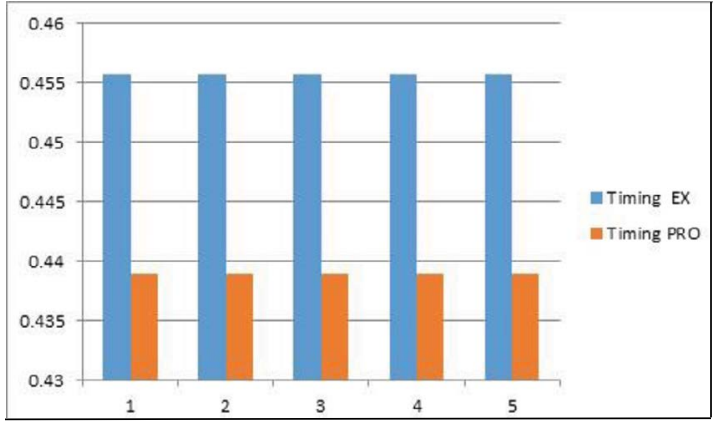

Figure 12. Time between previous method and existing method.

method. Figure 10-12 represents the overall comparison of distance, velocity and computation speed.

\section{Conclusion}

In this work pedestrian walk detection system is accomplished through multiple classifiers and the detection rates are viable with some of the existing methods to date in terms of both performance and running time. The tracking method is responsible for tracking the human activities by distinctive features. Histogram of gradient method is used for object tracking together with its increase in speed. Hence 
this method can be used for various applications that involve object tracking.

\section{References}

1. Wang C, Zhao C, Wang H. Self Similarity Based Zebra Crossing Detection for Intelligent Vehicle. The Open Automation and Control Systems Journal. 2015; 7:974-86. https://doi.org/10.2174/1874444301507010974

2. Alvarez JM, Gevers T, Le Cun Y, Lopez AM. Road scene segmentation form a single image. Springer-Verlag Berlin Heidelberg. 2012; p. 376-89.

3. Liu X, Zhang Y, Li Q. Automatic pedestrian crossing detection and impairment analysis based on mobile mapping system. ISPRS Annals of the Photogrammetry, Remote Sensing and Spatial Information Sciences. 2017; p. 18-22.
4. Viola P, Jones MJ, Snow D. Detecting pedestrians using patterns of motion and appearance. International Journal of Computer Vision. 2005; 63(2):153-61. https://doi. org/10.1007/s11263-005-6644-8

5. Ramzan H, Fatima B, Shahid AR, Ziauddin S, Safi AA. Intelligent pedestrian detection using optical flow and HOG. (IJACSA) International Journal of Advanced Computer Science and Applications. 2016; 7(9):408-17. https://doi.org/10.14569/IJACSA.2016.070955

6. Koester D, Lunt B, Stiefelhagen R. Zebra crossing detection from aerial imagery across countries. Springer International Publishing Switzerland. 2016; p. 1-8. https:// doi.org/10.1007/978-3-319-41267-2_5

7. Detection and intention prediction of pedestrians in zebra crossings. Available from: http://hh.diva-portal.org/smash/record.jsf?pid=diva2\%3A1246982\&ds wid $=1546940660347$. Date accessed 